\title{
How the Science of Memory Reconsolidation Advances the Effectiveness and Unification of Psychotherapy
}

\author{
Bruce Ecker ${ }^{1} \cdot$ Sara K. Bridges ${ }^{2,3}$
}

Published online: 22 April 2020

(c) The Author(s) 2020

\begin{abstract}
Memory reconsolidation research by neuroscientists has demonstrated the erasure of emotional learnings. This article reviews these historic findings and how they translate directly into therapeutic application to provide the clinical field with an empirically confirmed process of transformational change. Psychotherapists' early use of this new, transtheoretical knowledge indicates a strong potential for significant advances in both the effectiveness of psychotherapy and the unification of its many diverse systems. The erasure process consists of the creation of certain critical experiences required by the brain, and it neither dictates nor limits the experiential methods that therapists can use to facilitate the needed experiences. This article explains memory reconsolidation, delineates the empirically confirmed process, illustrates it in a case example of long-term depression, indicates the evidence supporting the hypothesis that this process is responsible for transformational change in any therapy sessions, describes the differing mechanisms underlying transformational change versus incremental change, and reports extensive clinical evidence that the basis and cause of most of the problems and symptoms presented by therapy clients are emotional learnings, that is, emotionally laden mental models, or schemas, in semantic memory.
\end{abstract}

Keywords Memory reconsolidation $\cdot$ Psychotherapy $\cdot$ Transformational change $\cdot$ Emotional learning $\cdot$ Mental model . Psychotherapy integration $\cdot$ Coherence therapy

\section{Introduction}

All mental health practitioners aim to help their clients permanently change unwanted behaviors, emotions, thoughts and somatizations. Yet, most practitioners also recognize that many of the changes they facilitate are incremental and susceptible to relapse, rather than transformational and life-altering. Various systems of psychotherapy do aim for and often produce profound, lasting change, ${ }^{1}$ but their accounts of how and why such change occurs are theoretical and metaphorical and differ greatly, as do their methods. This fragmented state of the psychotherapy field makes it difficult for clinicians to arrive at a clear and confident understanding of the fundamental mechanism and process

Bruce Ecker

bruce.ecker@coherenceinstitute.org

Coherence Psychology Institute, New York, NY, USA

2 The University of Memphis, Memphis, TN, USA

3 Coherence Psychology Institute, Albany, CA, USA of transformational change and why it can occur using so many different approaches.

If such knowledge were to emerge from an empirical, transtheoretical source, it would be invaluable for the psychotherapy field, driving advances in effectiveness and unification. This article proposes that such knowledge has already emerged from research by neuroscientists on memory reconsolidation (reviewed by, e.g., Agren 2014; Nader 2015; Reichelt and Lee 2013) and from the direct translation of those research findings into therapeutic application (Ecker 2011, 2015, 2018; Ecker et al. 2012; Ecker and Toomey 2008).

Memory reconsolidation (MR), discovered in the 1997-2000 period (reviewed by Riccio et al. 2006), is the brain's innate mechanism by which new learning experiences directly revise existing contents of memory acquired in prior learning. This updating of memory contents drives change at both the level of subjective experiencing and the level of neural encoding. MR is an experience-driven process of neurological change. It is a fundamental mechanism

\footnotetext{
1 Ten documentations of transformational change in diverse therapy systems are referenced online at https://bit.ly/15Z00HQ.
} 
that has been applied to many different types of memory using many different experiential processes, producing different types and degrees of change (reviewed by Ecker 2015, 2018).

The focus of this article is the capability of updating via MR to achieve profound unlearning that permanently nullifies a target emotional learning (reviewed by Clem and Schiller 2016). Many neuroscientists have termed that the "erasure" of the target learning (e.g., Agren et al. 2012; Clem and Huganir 2010; Kindt et al. 2009). The term erasure is used by researchers to mean that an acquired response is fully eliminated, that is, it can no longer be evoked into any detectable degree of behavioral, emotional or physiological expression by cues or contexts that previously did so. Functionally, it is as though the target emotional learning no longer exists in memory. Erasure of emotional learnings has been observed in studies with both animal and human subjects.

Such complete and lasting nullification of an acquired emotional response has never been produced by standard extinction procedures, which suppress the target learning only temporarily and usually only partially (Bouton 2004; Vervliet et al. 2013). Several studies have determined that memory reconsolidation and extinction are distinct phenomena at behavioral, neural, and molecular levels (Duvarci and Nader 2004; Duvarci et al. 2006; Merlo et al. 2014). Duvarci and Nader concluded, "Reconsolidation cannot be reduced down to facilitated extinction" (p. 9269).

The same well-defined, experience-driven process that has produced erasure in laboratory studies, when applied in psychotherapy to far more complex emotional learnings maintaining a wide range of clinical symptoms, has been observed to produce the same distinctive markers of erasure (Ecker 2015, 2018; Ecker et al. 2012), which now constitute transformational therapeutic change:

- the disappearance of a symptom (an unwanted behavior, affect, cognition and/or somatization),

- the disappearance of the symptom's accompanying emotional activation or distressed ego-state,

- the permanent, effortless persistence of those two changes.

Neural re-encoding through MR is the only mechanism and only type of neuroplasticity known to brain science that can produce those distinctive, unambiguous markers of erasure. In studies with human subjects, the markers of erasure are regarded by neuroscientists as evidence that MR and erasure have occurred (and the same markers are observed in animal studies in which analysis of brain tissue adds conclusive biochemical proof that MR and erasure have occurred).

As illustrated in the case example later in this article, the therapeutic target of change is a given symptom's underlying emotional learning, which is a schema, or mental model, that was learned long ago in emotionally intense experience and has been controlling behavior and/or state of mind from outside of awareness. The process of unlearning and erasing that schema or mental model thoroughly resolves and puts to rest a core, personal theme of emotional distress, and the markers of transformational change defined above immediately begin to appear.

\section{The Erasure of an Emotional Learning}

MR research by neuroscientists has demonstrated that an emotional learning is nullified by the following set of three experiences, which have therefore been termed the empirically confirmed process of erasure (ECPE) (Ecker 2018). Hundreds of MR research studies have used a vast range of different procedures and protocols to produce these experiences (reviewed by Ecker 2015, 2018), which means that what the brain requires for erasure of an emotional learning is not any particular external procedure, but rather the internal occurrence of these three subjective experiences, whatever may be the external procedures that create them. Therefore the ECPE does not dictate or favor the use of any particular therapeutic techniques, and psychotherapists are free to facilitate these critical experiences using any of the therapy field's vast array of experiential methods.

\section{Reactivated, Symptom-Generating Target Learning Experienced in Awareness}

This is the deliberate use of salient cues or contexts that reactivate the target emotional learning or schema underlying the client's presenting symptom or problem. For example, a woman in therapy for depression and absence of motivation was cued into reactivation of her lifelong schema that had newly come into awareness and was verbalized as, "Mom sees and knows everything I ever care about or do, and then takes over and takes away everything I ever care about or do, which feels devastating for me, and my only way to be safe from her pillaging is for me to care about nothing and do nothing." To assure that the schema is being directly accessed at its roots in the emotional learning and memory system and is not merely a cognitive insight, it is critically important that the emotions accompanying the reactivated schema are fully felt affectively and somatically while the schema also is cognized verbally and conceptually. Note that the schema is at core a mental model, from which are generated particular emotions, which in the example above would include helplessness, hopelessness, fear, desperation, despair, aloneness, and the deep pain of feeling used, pillaged and eclipsed in this way by her own mother. How that schema was found, brought 
into awareness, and then disconfirmed and unlearned is described in the case vignette in the next section.

\section{Experience of Mismatch/Prediction Error Destabilizes} the Target Learning's Neural Encoding

While the target schema is reactivated in awareness as described above, this is an additional, concurrent experience or knowing that contradicts what the client knows and expects according to the schema. This is termed a memory mismatch or prediction error experience by memory researchers. In response to this experience of the world differing from the target learning's expectations, the client's brain rapidly transforms the neural encoding of the target learning from its stable, consolidated state in long-term memory into a destabilized, de-consolidated, labile state, which is susceptible to being updated and re-encoded by any relevant new learning that may occur next. This destabilization, which requires and is triggered by the mismatch/prediction error experience, begins the reconsolidation process. ${ }^{2}$ The labile, destabilized condition persists for about $5 \mathrm{~h}$, widely termed the reconsolidation window, after which the neural encoding automatically reconsolidates, that is, it returns to a stable state in long-term memory. The case vignette below describes how a contradictory knowing was found for the schema of the depressed woman, creating the needed mismatch experience.

\section{Experience of Counter-Learning Drives Unlearning,} Nullification, Re-encoding and Replacement of Target Learning

This experience consists of just a few repetitions, during the rest of the therapy session, of the same mismatch experience created in the previous step. Each mismatch is a juxtaposition experience, in the sense that the client experiences both reality according to the target learning and a contradictory perception or knowing, with both in the same single field of awareness. Two or three repetitions of that juxtaposition experience serve as counterlearning that functions as an experiential disconfirmation of the target learning. Because the counter-learning is occurring while the encoding of the target learning is labile, the counter-learning rewrites and replaces the encoding of the target schema in memory. As a result,

\footnotetext{
2 At least thirty separate studies by neuroscientists, beginning with Pedreira, Pérez-Cuesta and Maldonado (2004), have demonstrated and confirmed that destabilization occurs not from target learning reactivation alone, but requires the two experiences of target learning reactivation and mismatch/prediction error. A list of those studies is online at https://bit.ly/2b8IbJH.
}

the target learning no longer exists in memory, so it cannot be reactivated and cause a relapse. The target learning is a model of the world in semantic memory, not an episodic memory of specific events and experiences; the latter is not erased. The unlearning of the target learning's version of reality is the profound resolution of a core emotional issue in the client's life, as noted earlier. ${ }^{3}$ Successful erasure of the target learning is then verified by observing the markers of transformational change beginning to appear immediately: the symptom(s) driven by the target learning cease to occur; the target learning itself, which previously was felt as a potent and horrible truth of the world, no longer feels true or real and is not reactivated by situations that formerly did so, eliminating a problematic, distressed ego state; and those changes persist effortlessly and permanently. If the same counter-learning occurs without first finding, reactivating and destabilizing the target learning (steps 1 and 2 above), the counter-learning only creates its own encoding separate from that of the target learning. In that case, the two learnings compete for control of behavior and state of mind, producing at best only incremental change that is prone to relapse when the emotionally more intense target learning becomes newly retriggered by current circumstances.

Prior to the detection of the MR phenomenon, over 60 years of research on extinction of acquired responses had led neuroscientists to conclude that the consolidation of an emotional learning into long-term memory was a one-time, irreversible process, and that the brain had no mechanism for de-consolidating, unlearning and erasing what had been learned. Therefore the discovery of MR was itself a transformational change and paradigm shift in the neuroscience of learning and memory.

The ECPE experiences defined above have been detected in previously published accounts of cases of transformational change from numerous different systems of therapy. ${ }^{4}$ For example, Ecker et al. (2012) provide demonstrations of

\footnotetext{
${ }^{3}$ Studies with animal and human subjects have appeared to show that a destabilized memory can also be erased by administering a chemical agent that disrupts the molecular process of restabilization or reconsolidation (e.g., Kindt et al. 2009). However, considerable controversy exists over both the interpretation and the uneven replicability of those results (e.g., Chalkia et al. 2019). With chemical erasure, there is no experience of unlearning, therefore no psychological resolution or mastering of core issues and no psychological growth, only symptom cessation. The authors view natural, endogenous erasure through experiential unlearning as being far preferable for general clinical practice because it has been observed to be effective for an extremely wide range of symptoms (described later in this article) and is accessible to all mental health practitioners.

${ }^{4}$ Documented online at https://bit.ly/15Z00HQ.
} 
ECPE detection in published cases of Accelerated Experiential Dynamic Psychotherapy (AEDP), Emotion-Focused Therapy (EFT), Eye Movement Desensitization and Reprocessing (EMDR), and Interpersonal Neurobiology (IPNB). The technique-independence of the ECPE is in that way demonstrated, revealing it to be a core process shared by diverse systems of psychotherapy that appear quite different on the level of technique. Thus knowledge of MR can serve as a framework of psychotherapy integration and unification (Ecker 2011, 2018; Ecker et al. 2012) in addition to guiding individual practitioners in the use of their preferred methods for producing transformational change with enhanced regularity.

It is widely recognized in the psychotherapy field that how clinicians conceptualize the process of change that they carry out does not necessarily identify all parts of the phenomenology that actually occurs in their sessions and is crucial in the process of change. For published cases in which the ECPE has subsequently been detected, it is instructive to compare the particular therapy system's account of how transformational change occurs with the ECPE phenomenology. For example, emotion-focused therapy (EFT) conceptualizes the process of change as "changing emotion with emotion" (Greenberg 2010, 2012): In the very context in which the client has been ruled by problematic emotion, an EFT practitioner facilitates a strong new experience of a different, healthy emotion.

By micro-analyzing an EFT case example by Greenberg (2010), Ecker et al. (2012, pp. 136-140) have shown that in carrying out that methodology, the component experiences of the ECPE occurred and immediately preceded the appearance of markers of transformational change: The client's lasting change of emotion followed the disconfirmation and nullification of a mental model that had been outside of awareness. Those ECPE experiences of schema disconfirmation and nullification were embedded in the therapy work, were not identified during the sessions or in the published case study, and, importantly, are not identified when the process of change is described as "changing emotion with emotion".

Nevertheless, therapists who adhere to EFT methodology are likely to carry out the ECPE and thereby achieve transformational change, for this reason: "By combining emotional reactivation of the target learning with a very different emotional experience of the original situation, a disconfirmation of [mental] model occurs implicitly, even though the attention of therapist and client may be focused on derivative emotion" (Ecker 2018, p. 80). As understood within the framework of MR and the ECPE, EFT is effective in producing transformational change because it is effective for nullifying the semantic structures (schemas, mental models) that underlie and generate the client's emotions, even though those semantic structures are not identified as the target of change in EFT's emotion-centric theory or methodology.

Furthermore, it is well established by MR research that the neurobiological mechanisms of destabilization and updating of memory contents do not inherently involve or require emotion whatsoever [reviewed and discussed in detail by Ecker (2015, pp. 25-29, 2018, pp. 79-80) and Ecker et al. (2015)]. Emotion almost always accompanies the ECPE in therapy not because emotion is required by the MR mechanism, but because the target learnings encountered in therapy almost always were formed in intensely emotional experiences, so they generate emotion when reactivated, and MR requires target learning reactivation (the first of the three experiences in the ECPE). Many studies have demonstrated the MR process with target learnings that involve no emotion, such as memory of a spatial arrangement of emotionally neutral objects, procedural memory of finger movements, or declarative/episodic memory of meaningless syllables. These research facts also mean that the MR mechanism is misrepresented by accounts that depict it as an inherently emotional or affective process (e.g., Lane et al. 2015; Stevens 2019; Welling 2012).

\section{Case Example}

To illustrate symptom generation by underlying emotional learnings and how such learnings are unlearned and erased through MR, producing transformational change, consider Tina, 33, who began her first therapy session by saying, "I've been feeling depressed and lousy for years. I have a black cloud around me all the time." She described a total absence of motivation, low energy, thorough social isolation, great difficulty doing her part-time, at-home work of writing grant proposals, and much self-denigration and self-pathologizing. She said, 'I' $m$ a vegetable. I' $m$ a worthless nothing that nobody could possibly find interesting." She had previously tried therapy, self-help groups, and Prozac, and was now taking Wellbutrin, but none of those had helped. More as an expression of hopelessness than curiosity she said, "I just don't know why I can't be happy." There was indeed a definite reason, but it was in her implicit emotional learnings, outside of awareness. Bringing that underlying emotional learning into direct, explicit awareness as a subjectively felt emotional truth would set it up as a target of change through MR. (For reviews of the neurobiology subserving the implicit and explicit memory systems, see, e.g., LaBar and Cabeza 2006; Squire 2004.)

Different therapy systems provide many different techniques for that retrieval from implicit to explicit knowing. In this case, the therapist was using Coherence Therapy (Bridges 2015; Ecker and Hulley 2019), which holds that a given symptom exists because, according to at least one 
adaptive emotional learning that is outside of conscious awareness, the symptom is compellingly necessary to have, even with the suffering entailed in having it. That is termed the principle of symptom coherence, and it guides the experiential process of finding and revealing the symptom's underlying emotional learning(s) or schema(s). Once revealed and brought into awareness, each schema generating the symptom is then subjected to the memory reconsolidation process of profound unlearning and erasure. Finally, observation of the markers of transformational change, verifying complete elimination of the symptom and its underlying core theme of emotional distress, is the last stage of Coherence Therapy for a given symptom. If the client also wants therapy to address other symptoms, the same process is carried out for them.

Here the therapist first enquired about Tina's experience in her family of origin. She described painful memories of several incidents, then in summary said flatly, "Saying what I'm really feeling or caring about gets me mowed down-so I don't go there."

The therapist then asked, "And how do you keep yourself from 'going there'?" That question arose from the therapist's assumption of symptom coherence, and it brought Tina's awareness deeper into an area where awareness had never gone. Her eyes darted around as she recognized and voiced with animation, "By being dead, apathetic, and telling myself I have nothing interesting to say!"

That began Tina's retrieval into awareness of emotional learnings that she had formed in response to the hostility that surrounded, endangered, and actually assaulted her in her family after almost every expression of her feelings, needs, thoughts and interests. Her autobiographical memory and conscious narratives already contained a great deal of what she had suffered and learned to expect in her family, but nothing about her own self-protective tactics that were urgently needed to avoid those assaults as much as possible. It was new, surprising awareness for her to recognize that for safety she had resorted to eliminating her own selfexpression by deadening herself.

With that shift from implicit to explicit knowing of why and how she had learned to protect herself, and from seeing for herself the fully coherent and adaptive nature of her solution to the problem of family hostility, her state of depression, lack of motivation and futility suddenly made deep sense to her in an entirely new way. Instead of viewing those symptoms as mystifying, out-of-control personal defects and pathology, she now recognized that they were urgently needed, purposeful, and effective tactics for keeping to a minimum the suffering that her family members were always ready to inflict.

That recognition of her agency and that de-pathologizing of her condition happened by becoming aware of her own emotional learning, and were, in themselves, significant therapeutic changes in her identity and model of herself.
The component experiences of the ECPE are apparent in how that change occurred: While she was viewing her depressed state of mind and behavior as pathology, she also experienced a very different, contradictory meaning that felt unmistakably true, and that juxtaposition of the two incompatible meaning-schemas was naturally repeated a few times in the rest of the session. Thus the ECPE was fulfilled and her pathologizing meaning for her depression was disconfirmed and erased. The retrieval of coherent emotional learnings in the initial, discovery phase of Coherence Therapy usually fulfills the ECPE in this way and has this dual effect of de-pathologizing the symptom and revealing the client's personal agency in producing it self-protectively. However, this would not be the only implementation of the ECPE in Tina's therapy.

At the start of her next session, Tina said she knew all along that in her family there was almost never any expression of love or affection, but it was new for her to recognize that in life with them she was "swimming with sharks." After five sessions of such work, the ECPE had also been applied to Tina's core belief that her family members' harsh negativity toward her was deserved and was caused by her own worthlessness. That mental model had been disconfirmed and replaced by the differently painful understanding that their behavior was caused by their own "emotional baggage" and their inability to be kind and loving. That work entailed feelings of hurt, anger, betrayal, and grief, yet her overall mood and appearance had lifted significantly, so she and the therapist agreed that she would schedule another session only if and when needed.

A few months later, she scheduled session six because, she explained, despite no longer being in a black cloud, a general apathy and lack of motivation had persisted. That report meant to the therapist that some part of her original emotional learning was still intact and perhaps was not yet fully retrieved into awareness.

To continue the discovery work, the therapist now guided Tina to imagine, just a little bit, how it will feel when she is no longer apathetic, actually has some interest and motivation in her own pursuits, and also mentions something about her pursuits to her parents. That is the discovery technique of symptom deprivation, often used in Coherence Therapy (Ecker and Hulley 2019). It is not a rehearsal for the client to then remain symptom-free after the session, which is explained to the client. Rather, any distress that develops due to being without the symptom during the exercise begins to reveal the emotional learning that the symptom is urgently necessary for avoiding that particular distress.

As Tina sampled the imaginal experience of mentioning her pursuits to her parents, she became quite uncomfortable in her chair and felt "pretty tense." The symptom deprivation exercise was working. A further implicit learning was emerging, and to bring it more fully into explicit expression, 
the therapist now invited Tina to let this sentence complete itself without pre-thinking: "If they know I'm doing things that matter to me-_." Tina said it once, and then again, and then very quietly she said: "She'll take it." She then became motionless, and after several seconds said loudly, in obvious amazement, "I erased myself!" As she then continued to find words for what had come into awareness, her voice and face became rageful: "She takes everything! She $\mathrm{f}^{* * * i n g}$ takes it all! So I've got to erase myself! She always, always makes it her accomplishment, not mine-so why should I be anything!"

Tina had retrieved into explicit awareness her emotional learning of both the ever-present danger of being pillaged and zeroed by her mother and the urgent need to thoroughly zero herself into apathy and blankness so that her mother will have no opportunity to do that to her ever again. What had seemed to be "depression" was now even more fully revealed to be Tina's own self-protective, adaptive tactic of making herself blank and "playing possum" to avoid that repeating ordeal.

This was major progress and another potent shift in Tina's experience. Even so, direct awareness of a symptom-generating emotional learning does not, in itself, necessarily produce a liberating nullification of it, as many experienced clinicians have observed. The ECPE maps out exactly what is needed for the brain to unlearn and nullify this deeply held material, producing transformational change: the experience of a contradictory knowing, in juxtaposition with the target learning. That had not yet occurred, and until it did, this material would remain in force.

Indeed, after several minutes of feeling both jubilant in her self-validation and empowered by her anger at her mother, Tina's state of mind slumped again as she grimly realized, and explained to the therapist, that her mother is still the same, so it feels as dangerous and scary as ever to care about anything and have any of her own interests and pursuits, or to even imagine revealing any such to her mother.

Hearing that, the therapist understood that there was still more to discover. Tina's continuing experience of raw vulnerability in relation to her narcissistic mother begged the question: What mental model of their relationship did Tina learn as a little girl that now, as an adult woman, keeps her feeling fully vulnerable to her mother? In Coherence Therapy parlance, what terms of attachment had her mother imposed that maintained such endangerment? The answer to that crucial question was in Tina's implicit learnings, and the therapist now had to find a way to elicit that material. Sentence completion, which had been effective earlier in the session, again fit the moment and the material. The therapist said, "Let this sentence complete itself, again without prethinking the ending, as we did earlier, ok? 'Mom can still take away whatever I might do or care about because-'. Say it yourself and just see what comes up to finish it."

Tina said, "Mom can still take away whatever I might do or care about because - anything I do or care about is right there in front of her, and she can take it away in one scoop." Tina then made a facial expression and gesture that meant, "Wasn't that really obvious all along?"

The implicit learnings of clients become obvious after they are discovered, but before that happens, they are deep, dark mysteries. For the therapist, what Tina had just described was a major revelation because it was now clear that her deadened state, and the whole schema maintaining it, were based entirely on this one core expectation, learned as a little girl, of being totally visible to her devouring mother. Reflecting back what Tina had revealed, the therapist said, "I see. Mom sees everything that you ever care about or do, and she takes over and takes away everything that she sees you ever caring about or doing, so your only way to be safe from that is for you to care about nothing and do nothing, so there's nothing that she sees."

Tina replied, "I never thought about it like that beforejust putting it in plain words like that—but that sums it up really well."

Having revealed that schema, the therapist, as a practitioner of the ECPE through Coherence Therapy, was now aiming to find contradictory knowledge that could disconfirm it. Tina's core expectation that anything she cares about or does is going to be visible to her mother was clearly a child's model of reality, and it might be readily disconfirmed by contrary adult knowledge already in Tina's possession if the two were brought into juxtaposition. In order to prompt Tina to find the needed contradictory knowledge, the therapist now gently said, "You feel just as vulnerable as ever to your mother taking things away. So tell me: In what ways do people keep other people from just reaching in and taking away things?".

That Socratic question was intended to guide Tina's attention into examining a crucial piece of her emotional learning that had always been outside of awareness and unthinkable. As Tina registered that question, her eyes blinked several times, she uttered a few fragmentary thoughts, then paused, furrowed her brow, and finally said, "It's as if there has been a 'no walls' rule all along. I think I've been obeying a 'no walls' rule." She then expressed amazement over seeing the possibility of "having walls" and keeping her personal affairs "behind walls" and totally unknown to her mother or others. Her seeing that such a possibility existed and was available to her was contradictory knowledge, and it came directly into juxtaposition with her mental model of her mother as all-seeing.

She again felt jubilant. This time, her elation was a marker of schema nullification and liberating change that persisted. The therapeutic process had gone beyond the retrieval and 
recognition of her emotional learnings to carrying out the ECPE once again, producing disconfirmation and a transformational change, as explained below. No further sessions were scheduled. In a follow-up phone talk two months later, Tina said her sixth session was a "major breakthrough" and laughed gleefully about having significant personal developments that she had kept fully private from her parents and siblings. She explained that the first month after her last session had been a rocky period of intense rage at her parents, but she now experienced only an occasional negative feeling toward them. Two years later, in another followup by telephone, Tina was nine months into a new career in computer programming and spoke about her future with enthusiasm. She said she was completely free of the black cloud and antidepressants. She added, "The work I did about my mom and her self-centeredness helped me a lot. I could step back-that's been really nice. Things are good, in many ways," she said with vitality in her voice, and then repeated, "Things are very good."

In Tina's final session, fulfillment of the ECPE is apparent from close examination of the moment-to-moment process. The target learning, now in awareness, was her mental model of her connection to her mother, a "no walls" model in which her inner and outer life were completely visible to mother and therefore always completely vulnerable to being plundered and pillaged by her. That mental model, formed in early childhood on the basis of myriad interactions between them, and then reinforced by myriad interactions all through growing up, was the very basis of the feeling of endangerment that made Tina's self-zeroing urgently necessary. She was solving the problem of selfhood robbery by having nothing to rob, a very costly solution that impoverished her mind and her life. The possibility of solving the problem by having walls, boundaries, and privacy from her mother had never existed for Tina. The therapist used a Socratic question to direct Tina's attention to discover that possibility for herself: "In what ways do people keep other people from just reaching in and taking away things?" Her adult knowings already held the answer to that question. With Tina's jolt of recognition that having walls is an option for her, the disconfirmation and erasure of the target learning were immediate and, therefore, so too was the ending of Tina's depression, lack of motivation, and social isolation, which had been necessitated and produced by nothing more than that emotional learning of helpless visibility. Two years of follow-up found that these transformational changes had persisted effortlessly, confirming erasure through the ECPE.

\section{Pragmatics of Carrying Out the ECPE}

The preceding case example shows that in order to create the transformative experiences defined by the ECPE, first the therapist must gather the needed materials through these preparatory steps (Ecker 2011; Ecker et al. 2012):

A. Symptom identification The therapist first clarifies with the client what to regard as the presenting symptom(s) the specific behaviors, circumstances, emotions, thoughts and/or somatics that the client wants to eliminate-and when they happen, that is, the cues and contexts that evoke or intensify them. This information is required so that step B can be pursued.

B. Retrieval of target learning The therapist then works to elicit into direct experience and explicit awareness the implicit emotional learning(s) or schema(s) underlying and producing the presenting symptom(s). This material, which is the target of change, must be richly felt affectively and somatically and also well delineated in words and concepts. Implicit emotional learnings seem at first to be blurry and elusive, but they prove to be well-defined and accessible. Step B is itself a multistep process in many cases, such as in the case example above, because emotional schemas can be multi-component constructions that are brought into awareness piece by piece. Knowledge of this material then guides step C.

C. Identification of disconfirming knowledge The therapist then works to find a vivid personal experience or knowing that contradicts what the client knows and expects according to the target schema. The contradictory material may be already part of the client's personal knowledge or may be created by a new experience. It will be used in carrying out the ECPE to create the mismatch that destabilizes the target learning and the counterlearning that nullifies and erases the target learning.

As soon as preparatory steps A, B and C are completed, the therapist can immediately facilitate ECPE experiences 1, 2 and 3 defined and illustrated earlier. Lastly, unlearning and erasure must be verified (in what has been labeled step $\mathrm{V}$, for verification) by observing the markers of transformational change: full disappearance of symptom(s), full disappearance of schema and ego-state reactivation, and effortless permanence of those changes.

Thus in practice the full process for facilitating the brain's mechanism of transformational change consists of the seven steps, ABC-123-V, named the Therapeutic Reconsolidation Process (TRP) by Ecker et al. (2012). Table 1 lists the steps of the TRP. Thus defined, the TRP is a versatile, techniqueindependent, transtheoretical methodology that enables psychotherapists to consistently replicate in their sessions 
Table 1 Steps of process for using memory reconsolidation in psychotherapy to induce transformational change (from Ecker et al. 2012)

\begin{tabular}{ll}
\hline Therapeutic reconsolidation process & \\
\hline I. Preparation phase & A. Symptom identification \\
& B. Retrieval of target learning (symptom-requiring schema) \\
& C. Identification of disconfirming knowledge \\
& 1. Reactivation of symptom-requiring schema (B) \\
II. Transformation phase (ECPE) & 2. Activation of disconfirming knowledge (C), mismatching \\
& symptom-requiring schema (B) \\
& 3. Repetitions of (B)-(C) pairing \\
III. Verification phase & V. Observations of: \\
& Emotional non-reactivation \\
& Symptom cessation \\
& Effortless permanence
\end{tabular}

the empirically confirmed process of erasure (ECPE) that neuroscientists used in laboratory studies to fully eliminate acquired emotional and behavioral responses.

One of the main messages sent to psychotherapists from MR research on the ECPE is that the profound unlearning of negative emotional learnings requires highly specific disconfirmation. That in turn sends the additional message that a major condition for success in carrying out the ECPE is the thorough retrieval of emotional learnings in detail (TRP step B), as illustrated in the case of Tina. Each therapy client has a unique emotional learning history and, therefore, unique and specific emotional learnings, which are not inferable from the client's presentation of problems and symptoms but can be revealed accurately through experiential methods.

Tina's depression was rooted in her intensely insecure and painful attachment relationship with her narcissistic mother. However, a secure attachment relationship with a therapist was not likely to produce transformational change, because her depression stemmed from a very specific feature within her attachment schemas, namely her non-conscious assumption of being always entirely visible to her mother. That specific construct was not likely to be either discovered or disconfirmed by a positive experience of a therapist's empathy, safety, alliance, or other "nonspecific common factors" widely believed responsible for nearly all therapeutic improvement. The unlearning and erasure of that one construct required creating an experience that contradicted and disconfirmed that construct specifically. The result was immediate transformational change.

There is no doubt that the nonspecific common factors are strongly conducive of therapeutic improvement, but their presence does not necessarily carry out the ECPE. A major implication of current brain science, supported by extensive clinical observations described earlier in this article, is that only if the ECPE occurs does erasure of emotional learning occur, producing transformational change. It is also a consistent observation by TRP practitioners that both schema nullification and symptom cessation occur immediately after the ECPE has been carried out. Similarly, as noted earlier it has been found consistently that when the markers of transformational change occur in therapy sessions conducted by clinicians who are not TRP practitioners, the ECPE in fact was carried out, though without the therapist's recognition. Collectively those observations imply that transformational change is caused directly by MR through the ECPE and is not a direct result of nonspecific common factors that had been present all through the sessions prior to completion of the ECPE.

In some cases, the client's experience of the therapist's empathy, caring or kindness itself serves as the disconfirmation of a symptom-generating schema, fulfilling the ECPE. This again demonstrates the necessity of the ECPE for transformational change to occur. The MR/ECPE framework refutes, empirically and decisively, the assertion of nonspecific common factors theory (e.g., Duncan et al. 2010) that a specific process can never be a reliable cause of powerful therapeutic effectiveness (discussed by, e.g., Ecker 2013, 2018; Ecker et al. 2012, pp. 154-155).

The ECPE refines and advances our understanding of what constitutes a "corrective experience" (Ecker 2015, 2018) by making it explicit that creating the needed, healthy experience that was missing in the client's life is not enough, by itself, to produce deep, lasting change. Rather, the brain requires juxtaposition of that needed, healthy experience with concurrent reactivation of the client's learned, negative emotional schema, so that the latter is disconfirmed and nullified by the former. The ECPE in that way solves the mystery of why lasting change is not produced by many attempted corrective experiences that feel deep, meaningful and satisfying to the client in the moment.

Fully retrieving negative emotional learnings into awareness feels counter-intuitive for some therapists because in doing so, the process is heading straight toward the source of all the trouble, rather than away from it. Many therapists conduct therapy as guided by their counteractive reflex (Ecker et al. 2012), an almost universal tendency to disconnect from, suppress, oppose and fix unwanted states of mind and behaviors by focusing on building up preferred states of mind and behaviors. That strategy of counteracting produces only incremental, unstable change, as a rule (Ecker 
and Toomey 2008; Toomey and Ecker 2009). It does produce new learning, but that new learning forms separately from, and competes with, the powerful emotional learning maintaining the client's symptoms, as noted earlier. With experiences of success in carrying out the TRP/ECPE, a therapist knows and feels that heading straight toward the symptom's underlying emotional learnings is a direct path toward a liberating therapeutic breakthrough, as can be seen in the case example of Tina.

Various complications can and do occur in carrying out the TRP. For example, any of its steps may be met with resistance. If the symptom's underlying emotional learning involves traumatic memory, the discovery work in TRP step B may encounter resistance until the therapist sensitively adjusts the pace and manner of the work to be tolerable for the client. Most experienced clinicians are familiar with that kind of resistance. A type of resistance that is unique to the TRP/ECPE occurs in response to step 3, the counter-learning that would disconfirm, nullify and erase the target schema if no resistance occurs. Describing such resistance to schema nullification, Ecker et al. (2012, p. 62) explain, "The dissolution of personal constructs...is allowed to proceed, or not, depending on whether the emotional results feel tolerable to the client's emotional brain in all areas, both consciously and unconsciously; the nature of the process is not simply mechanistic or neurological." Ecker (2018, p. 31) further observes,

The brain's implicit predictive capability proves to be remarkably astute regarding unacceptable adjustments entailed by a particular schema losing realness and being decommissioned. The schema simply remains in force (continues to feel compellingly real and potent) despite well-crafted juxtaposition experiences being guided. That persistence of the schema is the therapist's indicator that there is some blocking contingency that now must be sensitively brought into awareness, recognized and addressed. When the client arrives at feeling that the (now consciously) anticipated difficulty is workable, the therapist repeats the juxtaposition experiences of Step 3, and schema nullification now is allowed to occur.

For a detailed case example of this kind of resistance and how it is dispelled, see Ecker et al. (2012, pp. 77-86). In our case example above, Tina's change of self-protective tactic from total self-zeroing to total privacy from her mother entailed a bold new degree of autonomy and a sharp loss of her original attachment pattern with her mother. If Tina's implicit knowledge system anticipated that those shifts were going to bring intolerable aloneness and anxiety, her non-conscious, anticipatory terror would have shut down her experiential faculties, preventing the new possibility of "having walls" from feeling real or available to her, so the juxtaposition experience would have been blocked and her transformational breakthrough would not have occurred at that point.

Another type of complication consists of the client having more than one emotional learning or schema generating the same symptom. In such cases of multiple causation, the symptom ceases completely only after all of its underlying schemas have been found and then disconfirmed and dissolved by the ECPE. For example, if Tina's depression had been also an expression of suppressed despair and grief over never receiving warmth, love, or caring understanding, that too would need to have been resolved before her depression could disappear (and that too would entail the same ECPE process of awareness and disconfirmation of schemas, in this instance schemas forbidding and/or invalidating feelings of despair and grief, and therefore requiring their suppression). The existence of more than one symptom-generating schema is indicated when the first-found schema has been decisively nullified but the symptom continues to occur.

As noted, an emotional learning is at core a mental model in some area of experience that entailed strong emotion. In many attachment-oriented frameworks of psychotherapy, the client's learned "internal working models" of self and others are viewed as the basis of problematic behaviors and states of mind (e.g., Brown and Elliott 2016; Teyber and Teyber 2016). Certainly attachment experiences and learnings are a major domain of human life, but there are also numerous other domains in which people learn potent, symptomgenerating mental models. Ecker et al. (2012) have identified existential, social, artistic, sexual, athletic, and spiritual domains, in addition to the attachment domain, and emphasize that therapists are optimally positioned for accurate, efficient retrieval of clients' schemas by not assuming that the client's underlying schema is about attachment. The actual domain of the schema will be clearly apparent from the material that emerges through clean, thorough, experiential discovery work. "Like different rivers converging to one delta to enter the ocean, all of the domains of learning... converge to the same locus of influence, namely the contents of the individual's emotional implicit memory" (Ecker et al. 2012, p. 96).

\section{The Role of Memory in Symptom Production}

MR in general, and the ECPE in particular, create change by updating the existing content of learning and memory. Therefore, the usefulness of the TRP/ECPE for psychotherapy depends upon the extent to which learning and memory are the basis of clinical symptoms, as distinct from other types of causation, such as neurochemical imbalances due to genetics or inherited epigenetics, or inborn temperament. Extensive clinical observations reviewed by Ecker (2018) 
Table 2 Clinical symptoms observed to be ended by applying the therapeutic reconsolidation process (from Ecker 2018, p. 31)

\begin{tabular}{ll}
\hline Symptoms ended $^{\text {a }}$ & \\
\hline Aggressive behavior & Food/eating/weight problems \\
Agoraphobia & Grief and bereavement problems \\
Alcohol abuse & Guilt \\
Anger and rage & Hallucinations \\
Anxiety & Indecision \\
Attachment-pattern-based behaviors and distress & Low self-worth, self-devaluing \\
Attention deficit problems & Panic attacks \\
Codependency & Perfectionism \\
Complex trauma symptomology & Procrastination/inaction \\
Compulsive behaviors of many kinds & Psychogenic/psychosomatic pain \\
Couples' problems of conflict/communication/closeness & PTSD symptoms \\
Depression & Sexual problems \\
Family and child problems & Shame \\
Fidgeting & Underachieving \\
& Voice/speaking/singing problems \\
\hline
\end{tabular}

${ }^{a}$ An online bibliography of published case examples indexed by symptom is available at https://bit. ly/2tKXdyX indicate that MR is applicable for a very wide range of clinical symptoms. Table 2 lists clinical symptoms that TRP practitioners have observed to cease permanently as soon as the ECPE has been carried out and one or more specific schemas have been nullified and no longer feel real. The extensive list implies that the vast majority of therapy clients' symptoms are generated by emotional learnings that prove to be accessible, well defined, and nullifiable through disconfirmation in the ECPE.

Two main types of memory are important in symptom production: memory of particular personal experiences, or episodic memory, which is not merely the cognitive, factual memory of what happened but rather the living memory of the subjective experience of the event; and memory of what has been learned about the nature and behavior of the world (including people and oneself), or semantic memory. Symptom-generating semantic memory consists of nonverbal yet well-defined, schematic mental models that operate from outside of awareness to generate problematic behavior, state of mind, and somatic conditions, as shown in the case example of Tina. A review of the nature, mutual interaction, and clinical phenomenology of episodic and semantic memory is beyond the scope of this article; for such review, see, for example, Ecker $(2015,2018)$ and Lane et al. (2015). Here, the noteworthy point is that in psychotherapy, episodic memory serves as a portal of access to semantic knowledge. Transformational change is the result of an unlearning and nullification of some unit of semantic knowledge, even when it may seem that such change resulted from a therapeutic process focused on episodic memory. For example, Tina's in-session revisiting of various specific past experiences in her family served for consciously recognizing the implicit schematic knowledge she had formed from those experiences, specifically her implicit knowledge of the danger of family members' hostility to her every self-expression, the danger of her mother's pillaging of her every interest and motivation, and her mother's "no walls" rule, plus her implicit knowledge of how to protect herself from all of that by thoroughly deadening her own capacity for interest, motivation, and self-expression.

The same is apparent in cases of successful dispelling of post-traumatic symptomology: Symptoms stemming from trauma cease to occur as soon as their underlying semantic knowledge is disconfirmed, unlearned and nullified by the ECPE (for detailed case examples, see Ecker 2018, pp. 33-50 and Ecker et al. 2012, pp. 86-91). That profound change in semantic memory causes no change in episodic memory of what happened in the original traumatic experience (as shown by Kindt et al. 2009), which confirms that the symptoms were maintained by semantic memory and not by episodic memory. No ethical problems arise with erasure of semantic memory because such erasure is the obsolescence of a mental model of the world that was construed on the basis of particular experiences, which causes no impairment of autobiographical and episodic memory of those experiences.

The potent qualities of traumatic memory are generated by the distinctive features of the semantic emotional schemas formed in response to trauma, such as:

- a model of oneself as being utterly helpless, and therefore gravely vulnerable, in a particular type of endangering situation, generating hypervigilance, avoidance behaviors, and terror/panic or dissociation when any slightest sign of that type of situation is perceived to be appearing,

- a model of oneself as incapable of enduring or surviving the conscious experience of a suppressed traumatic 
memory, generating suppression of the episodic memory, avoidance of situations that would be reminders of it, flashbacks, and recurrent nightmares,

- a model of oneself as triggerable into panic or terror by certain situations (the fear-of-fear condition), generating anxious hypervigilance and avoidance behaviors,

- an attributed meaning about self, others, or the world that is intensely distressing, generating chronic retriggering of certain emotions, attitudes and behaviors. ${ }^{5}$

This article focuses on applying MR to target memory material consisting of semantic memory, because that can produce transformational change, the most effective therapeutic outcome. However, as noted earlier, MR can be applied to any type of memory, including episodic memory, through any process that reactivates the target memory, then destabilizes the reactivated target memory by creating an experience of prediction error (memory mismatch), and then creates a new learning experience that is encoded into the destabilized target memory. For applying MR directly to episodic memory of traumatic experiences, the process consists of reactivating the target traumatic memory and concurrently creating an emotionally positive experience, so that the encoding of the target episodic memory becomes conjoined with the encoding of the new episodic memory of the positive experience. Any subsequent retriggering of the traumatic memory would then also activate the conjoined positive memory, thereby partially limiting the traumatic memory's emotional potency, intrusions into awareness, and other problematic effects on behavior, body, and/or state of mind.

That strategy of change is known as episodic memory interference (e.g., Bryant and Datta 2019; for review of laboratory studies, see Scully et al. 2017; for detailed discussion of clinical examples, see Ecker 2018). That strategy cannot erase the target episodic memory of trauma because it cannot disconfirm the client's personal knowledge of having had the experiences in that episodic memory. Furthermore, the negative emotional valence of traumatic memories is significantly stronger than the positive valence of any pleasurable

\footnotetext{
${ }^{5}$ For a case example of this type, see Ecker (2018, pp. 33-37).

${ }^{6}$ Use of psychedelic drugs has been found to enhance effectiveness of therapy for PTSD and addictions, and in explanation, various hypotheses have been advanced, one of which is the episodic memory interference effect: Psychedelics possibly create positive, pleasurable experiences of sufficient potency to outweigh the negative emotional valence of the target traumatic memory. This use of psychedelics has risks and ethical problems, would be safe for only the most stable therapy clients, and could not be utilized by a large majority of licensed mental health practitioners.
}

or comforting experience that can be created in therapy sessions, as a rule, so it seems probable that episodic memory interference is inherently limited to producing moderate reduction of post-traumatic symptoms. ${ }^{6}$

With a partial reduction of symptoms, MR is not the only possible explanatory mechanism, because some type of counteractive, competitive change not due to MR could also be hypothesized. Only when symptoms and schema or ego-state reactivations disappear completely and lastingly, indicating erasure of a semantic emotional learning, is MR the only viable explanatory mechanism, because only MR can produce erasure. ${ }^{7}$

Also, episodic memory interference can not be expected to alleviate post-traumatic symptoms generated by the semantic emotional schemas learned from the traumatic experience(s), because such schemas function independently of episodic memory of the experiences that formed them (Ecker 2018). Given that focusing trauma therapy on disconfirming and erasing semantic schemas can be significantly more effective than episodic memory interference, there is reason to question why the latter approach is widely explored. In the authors' experience, it is rare for research or clinical articles on episodic memory interference to note the existence or clinical importance of semantic memory, which possibly indicates that the pervasive, symptom-producing role of semantic memory is widely unrecognized.

Lastly, an understanding of semantic emotional learning and memory according to current neuroscience calls into question some of the clinical field's pathologizing terminology. Anything learned in the presence of strong emotion is encoded under the influence of hormones that produce extremely durable memory in subcortical memory networks (McGaugh 1989; McGaugh and Roozendaal 2002). The brain evolved so that such learnings do not fade over time and are not unlearned by standard extinction procedures (Bouton 2004; LeDoux et al. 1989). Thus the long-term persistence and retriggering of implicit, adaptive emotional learnings, such as those of Tina in our case example, are the proper functioning, not the malfunctioning, of the brain's implicit memory system. In that way the brain science of implicit, adaptive emotional learning and unlearning contradicts the conventional view in the clinical field that the core beliefs and schemas underlying symptoms are irrational, maladaptive or pathogenic, and shows, rather, that they are entirely coherent and adaptive within the individual's life experiences and learnings.

\footnotetext{
${ }^{7}$ Neuroscientists have identified various potential obstacles to the successful clinical application of MR research findings, but the clinical use of MR for erasure, achieving the decisive, transformational effects of erasure, avoids those problems. For extended discussion of these issues, see Ecker (2018, pp. 51-60).
} 


\section{Conclusion}

As described and shown in the preceding sections, from the perspective of memory reconsolidation, the existence of a therapy client's symptomatology indicates the presence, outside of awareness, of distress-laden emotional learnings (schemas, mental models) generating the symptoms with adaptive intent; and the optimal process of psychotherapy consists of guiding the profound unlearning of the symptomgenerating emotional learnings, nullifying and erasing them via the memory reconsolidation process. That perspective has been found applicable to a wide range of symptomatology. However, the clinician must be alert to recognize symptoms that are not based in learning and memory and therefore cannot be dispelled by MR, such as Asperger's syndrome or depression due to hypothyroidism.

Understanding change in terms of memory reconsolidation does not necessarily require a therapist to make any change in the methods normally used in sessions, though it is likely to prompt rewarding adjustments in how familiar methods are used. It is also likely to make new sense of unfamiliar methods, expanding a therapist's repertoire of options for responding effectively to clients and their material.

As the importance of MR for psychotherapy has continued to become more widely recognized by clinicians, its operation and use have been described by clinical authors in a variety of ways, some of which wander far from any correspondence to the actual MR research findings and are, in certain cases, actually precluded by the empirical findings (documented by Ecker 2015, 2018; Ecker et al. 2015). Therefore it warrants noting here that the term "reconsolidation" is not merely a neuroscientific synonym for "change" and not merely a neuroscientific endorsement that lasting change is possible, leaving clinicians free to define how MR operates based on favored psychological theory, or psychotherapy outcome research, or clinical observations. Rather, "reconsolidation" denotes a particular process consisting of specific steps on both the experiential and the neurological level. Any claim of using MR in therapy depends for validity on showing that the therapeutic process carried out fulfills the empirically confirmed requirements of the brain for recruiting MR.

Clinicians' descriptive terminology also wanders easily away from scientific accuracy: What reconsolidates is a neural encoding of memory contents, not an affect, not a cognition, not a behavior. The neural encoding of the target memory material undergoes a biochemical transition first from a stable state in long-term, consolidated memory to an unstable, deconsolidated state of vulnerability to modification, followed by neural re-encoding driven by new learning, and then back into a stable, consolidated state. Changes in affect, cognition, or behavior are then observed as a result of that re-encoding and its reconsolidation. Thus phrases such as "affect reconsolidation" or "reconsolidated behavior" embody a fundamentally incorrect conceptualization and can create an inaccurate understanding of the MR mechanism of change.

Psychotherapy outcome researchers have all along regarded merely a partial, modest, incremental improvement as successful therapy (Shedler 2015; Wampold and Imel 2015). Some outcome studies have reported therapeutic effects that were represented as successful outcome because they met technical criteria for being statistically significant, when actually the effects were too small to be clinically significant (Lambert and Ogles 2009). As a result of such research practices, the clinical field's standard of effectiveness has been quite low. Suddenly, MR research confronts the clinical field with empirical proof of a built-in mechanism of a far more potent type of change, transformational change. This potentially heralds a fundamental reshaping of the landscape of the psychotherapy field. Knowledge of MR confirms that the mind and brain have a natural, native capability for profound, liberating therapeutic change, so the job of the therapist is to facilitate skillfully that innate capability of each person to get free from the tenacious grip of negative emotional learnings.

There is immense value for the psychotherapy field in now having transtheoretical, empirical knowledge of the brain's mechanism of change that comes from outside of the psychotherapy field. Perhaps only in this way can the field find robust, universally acceptable unification that escapes the conundrum of the field's entrapment in inconclusive theorizing that is perpetually contested among parochial factions. As this article has emphasized, many existing theories and methodologies of psychotherapy do successfully guide clinicians to produce transformational change some of the time. Under the meta-framework of memory reconsolidation, there is no need to challenge any of those theories or methodologies, because it now becomes possible to see how and why each system works when it works, and each becomes a unique candidate for how to facilitate the Therapeutic Reconsolidation Process in an optimal manner for a particular client at a particular moment. The authors are confident that importing accurate knowledge of MR will bring a prolonged golden era of unprecedented effectiveness and unification to the psychotherapy field.

Acknowledgements The authors thank Dr. Melissa Reading and Robin Ticic for reading drafts of this article and enhancing its clarity.

\section{Compliance with Ethical Standards}

Conflict of interest Bruce Ecker and Sara K. Bridges are unpaid as the directors of the Coherence Psychology Institute, which provides training in the psychotherapeutic framework described in this article. 
Bruce Ecker and Sara K. Bridges declare that they have no conflicts of interest.

Ethical Approval This article does not contain any studies with human participants or animals performed by any of the authors.

Open Access This article is licensed under a Creative Commons Attribution 4.0 International License, which permits use, sharing, adaptation, distribution and reproduction in any medium or format, as long as you give appropriate credit to the original author(s) and the source, provide a link to the Creative Commons licence, and indicate if changes were made. The images or other third party material in this article are included in the article's Creative Commons licence, unless indicated otherwise in a credit line to the material. If material is not included in the article's Creative Commons licence and your intended use is not permitted by statutory regulation or exceeds the permitted use, you will need to obtain permission directly from the copyright holder. To view a copy of this licence, visit http://creativecommons.org/licenses/by/4.0/.

\section{References}

Agren, T. (2014). Human reconsolidation: A reactivation and update. Brain Research Bulletin, 105, 70-82. https://doi.org/10.1016/j. brainresbull.2013.12.010.

Agren, T., Engman, J., Frick, A., Björkstrand, J., Larsson, E. M., Furmark, T., et al. (2012). Disruption of reconsolidation erases a fear memory trace in the human amygdala. Science, 337, 1550-1552. https://doi.org/10.1126/science.1223006.

Bouton, M. E. (2004). Context and behavioral processes in extinction. Learning and Memory, 11, 485-494. https://doi.org/10.1101/ $\operatorname{lm} .78804$.

Bridges, S. K. (2015). Coherence therapy: The roots of problems and the transformation of old solutions. In H. E. A. Tinsley, S. H. Lease, \& N. S. Giffin Wiersma (Eds.), Contemporary theory and practice in counseling and psychotherapy (pp. 353-380). Thousand Oaks/London: Sage Publications.

Brown, D. P., \& Elliott, D. S. (2016). Attachment disturbances in adults: Treatment for comprehensive repair. New York: W. W. Norton \& Company.

Bryant, R. A., \& Datta, S. (2019). Reconsolidating intrusive distressing memories by thinking of attachment figures. Clinical Psychological Science. https://doi.org/10.1177/2167702619866387.

Chalkia, A., Weermeijer, J., Van Oudenhove, L., \& Beckers, T. (2019). Acute but not permanent effects of propranolol on fear memory expression in humans. Frontiers in Human Neuroscience, 13, 51. https://doi.org/10.3389/fnhum.2019.00051.

Clem, R. L., \& Huganir, R. L. (2010). Calcium-permeable AMPA receptor dynamics mediate fear memory erasure. Science, 330, 1108-1112. https://doi.org/10.1126/science.1195298.

Clem, R. L., \& Schiller, D. (2016). New learning and unlearning: strangers or accomplices in threat memory attenuation? Trends in Neuroscience, 39(5), 340-351. https://doi.org/10.1016/j. tins.2016.03.003.

Duncan, B. L., Miller, S. D., Wampold, B. E., \& Hubble, M. A. (Eds.). (2010). The heart and soul of change: Delivering what works in therapy (2nd ed.). Washington, DC: American Psychological Association. https://dx.doi.org/10.1037/12075-000

Duvarci, S., \& Nader, K. (2004). Characterization of fear memory reconsolidation. Journal of Neuroscience, 24, 9269-9275. https ://doi.org/10.1523/JNEUROSCI.2971-04.2004.

Duvarci, S., Mamou, C. S., \& Nader, K. (2006). Extinction is not a sufficient condition to prevent fear memories from undergoing reconsolidation in the basolateral amygdala. European
Journal of Neuroscience, 24, 249-260. https://doi.org/10.111 $1 / \mathrm{j} .1460-9568.2006 .04907 . x$.

Ecker, B. (2011, January 13). Reconsolidation: A universal, integrative framework for highly effective psychotherapy [Web log post]. Retrieved September 10, 2019, from https://www.mentalhelp.net/ blogs/reconsolidation-a-universal-integrative-framework-for-highl y-effective-psychotherapy/.

Ecker, B. (2013). Nonspecific common factors theory meets memory reconsolidation: A game-changing encounter? The Neuropsychotherapist, 2, 134-137. https://doi.org/10.12744/tnpt(2)134-137.

Ecker, B. (2015). Memory reconsolidation understood and misunderstood. International Journal of Neuropsychotherapy, 3, 2-46. https://doi.org/10.12744/ijnpt.2015.0002-0046.

Ecker, B. (2018). Clinical translation of memory reconsolidation research: Therapeutic methodology for transformational change by erasing implicit emotional learnings driving symptom production. International Journal of Neuropsychotherapy, 6(1), 1-92. https://doi.org/10.12744/ijnpt.2018.0001-0092.

Ecker, B., \& Hulley, L. (2019). Coherence therapy practice manual and training guide. San Francisco, CA: Coherence Psychology Institute. Online: https://www.coherencetherapy.org/resources/ manual.htm

Ecker, B., Hulley, L., \& Ticic, R. (2015). Minding the findings: Let's not miss the message of memory reconsolidation research for psychotherapy. Behavior and Brain Sciences, 38, e7. https://doi. org/10.1017/S0140525X14000168.

Ecker, B., Ticic, R., \& Hulley, L. (2012). Unlocking the emotional brain: Eliminating symptoms at their roots using memory reconsolidation. New York: Routledge.

Ecker, B., \& Toomey, B. (2008). Depotentiation of symptom-producing implicit memory in coherence therapy. Journal of Constructivist Psychology, 21, 87-150. https://doi.org/10.1080/1072053070 1853685.

Greenberg, L. S. (2010). Emotion-focused therapy: A clinical synthesis. Focus, 8, 32-42. Online: https://focus.psychiatryonline.org/ cgi/reprint/8/1/32.

Greenberg, L. (2012). Emotions, the great captains of our lives: Their role in the process of change in psychotherapy. American Psychologist, 67, 697-707. https://doi.org/10.1037/a0029858.

Kindt, M., Soeter, M., \& Vervliet, B. (2009). Beyond extinction: Erasing human fear responses and preventing the return of fear. Nature Neuroscience, 12, 256-258. https://doi.org/10.1038/nn.2271.

LaBar, K. S., \& Cabeza, R. (2006). Cognitive neuroscience of emotional memory. Nature Reviews Neuroscience, 7(1), 54-64. https ://doi.org/10.1038/nrn1825.

Lambert, M. J., \& Ogles, B. M. (2009). Using clinical significance in psychotherapy outcome research: The need for a common procedure and validity data. Psychotherapy Research, 19(4-5), 493-501. https://doi.org/10.1080/10503300902849483.

Lane, R. D., Ryan, L., Nadel, L., \& Greenberg, L. (2015). Memory reconsolidation, emotional arousal and the process of change in psychotherapy: New insights from brain science. Behavioral and Brain Sciences, 38, e1. https://doi.org/10.1017/S0140525X1 4000041.

LeDoux, J. E., Romanski, L., \& Xagoraris, A. (1989). Indelibility of subcortical emotional memories. Journal of Cognitive Neuroscience, 1, 238-243. https://doi.org/10.1162/jocn.1989.1.3.238.

McGaugh, J. L. (1989). Involvement of hormonal and neuromodulatory systems in the regulation of memory storage. Annual Review of Neuroscience, 2, 255-287. https://doi.org/10.1146/annur ev.ne.12.030189.001351

McGaugh, J. L., \& Roozendaal, B. (2002). Role of adrenal stress hormones in forming lasting memories in the brain. Current Opinions in Neurobiology, 12, 205-210. https://doi.org/10.1016/S0959 -4388(02)00306-9. 
Merlo, E., Milton, A. L., Goozée, Z. Y., Theobald, D. E., \& Everitt, B. J. (2014). Reconsolidation and extinction are dissociable and mutually exclusive processes: Behavioral and molecular evidence. The Journal of Neuroscience, 34, 2422-2431. https://doi. org/10.1523/jneurosci.4001-13.2014.

Nader, K. (2015). Reconsolidation and the dynamic nature of memory. Cold Spring Harbor Perspectives in Biology, 7, a021782. https:// doi.org/10.1101/cshperspect.a021782.

Pedreira, M. E., Pérez-Cuesta, L. M., \& Maldonado, H. (2004). Mismatch between what is expected and what actually occurs triggers memory reconsolidation or extinction. Learning \& Memory, 11, 579-585. https://doi.org/10.1101/lm.76904.

Reichelt, A. C., \& Lee, J. L. C. (2013). Memory reconsolidation in aversive and appetitive settings. Frontiers of Behavioral Neuroscience, 7, 1-18. https://doi.org/10.3389/fnbeh.2013.00118.

Riccio, D. C., Millin, P. M., \& Bogart, A. R. (2006). Reconsolidation: A brief history, a retrieval view, and some recent issues. Learning \& Memory, 13, 536-544. https://doi.org/10.1101/lm.290706.

Scully, I. D., Napper, L. E., \& Hupbach, A. (2017). Does reactivation trigger episodic memory change? A meta-analysis. Neurobiology of Learning and Memory, 142(Part A), 99-107. https://doi. org/10.1016/j.nlm.2016.12.012.

Shedler, J. (2015). Where is the evidence for "evidence-based" therapy? The Journal of Psychological Therapies in Primary Care, 4(1), 47-59. https://doi.org/10.1016/j.psc.2018.02.001.

Squire, L. (2004). Memory systems of the brain: A brief history and current perspective. Neurobiology of Learning and Memory, 82, 171-177. https://doi.org/10.1016/j.nlm.2004.06.005.

Stevens, F. L. (2019). Affect regulation and affect reconsolidation as organizing principles in psychotherapy. Journal of Psychotherapy Integration, 29(3), 277-290. https://doi.org/10.1037/int0000130.

Teyber, E., \& Teyber, F. H. (2016). Interpersonal process in therapy: An integrative model (7th ed.). Pacific Grove, CA: Brooks/Cole.
Toomey, B., \& Ecker, B. (2009). Competing visions of the implications of neuroscience for psychotherapy. Journal of Constructivist Psychology, 22, 95-140. https://doi.org/10.1080/1072053080 2675748.

Vervliet, B., Craske, M. G., \& Hermans, D. (2013). Fear extinction and relapse: state of the art. Annual Review of Clinical Psychology, 9, 215-248. https://doi.org/10.1146/annurev-clinpsy-05021 2-185542.

Wampold, B. E., \& Imel, Z. E. (2015). The great psychotherapy debate: The evidence for what makes psychotherapy work. New York: Routledge.

Welling, H. (2012). Transformative emotional sequence: Towards a common principle of change. Journal of Psychotherapy Integration, 22, 109-136. https://doi.org/10.1037/a0027786.

Publisher's Note Springer Nature remains neutral with regard to jurisdictional claims in published maps and institutional affiliations.

Bruce Ecker is co-originator of Coherence Therapy, co-director and co-founder of the Coherence Psychology Institute, and coauthor of Unlocking the Emotional Brain: Eliminating Symptoms at Their Roots Using Memory Reconsolidation; the Coherence Therapy Practice Manual \& Training Guide; and Depth Oriented Brief Therapy. How transformational change occurs is his interest.

Sara K. Bridges is Associate Professor at the University of Memphis, coeditor of the five-volume series Studies in Meaning, author of the Coherence Therapy chapter in the graduate text, Contemporary Theory and Practice of Counseling and Psychotherapy (Sage), and co-director of the Coherence Psychology Institute. 